\title{
Prescription Writing Skills of Doctors Practicing in Dhule City of Maharashtra State: A Cross Sectional Survey
}

\author{
Harish C. Jadhavi ${ }^{1}$, Arun S. Dodamani ${ }^{1}$, Prashanthkumar Vishwakarma ${ }^{1}$, Girija A. Dodamani ${ }^{2}$, Mahesh \\ R. Khairnar ${ }^{3^{*}}$, Rahul G. Naik ${ }^{4}$ \\ ${ }^{1}$ Department of Public Health Dentistry, ACPM Dental College and Hospital, Dhule, INDIA. \\ ${ }^{2}$ Department of Prosthodontics, ACPM Dental College and Hospital, Dhule, INDIA. \\ ${ }^{3}$ Department of Public Health Dentistry, Bharati Vidyapeeth Deemed University Dental College and Hospital, Sangli, INDIA. \\ ${ }^{4}$ Department of Public Health Dentistry, ACPM Dental College and Hospital, Dhule, INDIA.
}

\begin{abstract}
Objective: Due concern growing regarding incomplete or illegible prescriptions, the present survey was undertaken to assess the prescription writing skills of doctors practicing in Dhule city. Material and methods: 286 prescriptions dispensed by various doctors were collected for 5 consecutive days from 5 randomly selected pharmacy shops in Dhule city, Maharashtra state, India. The prescriptions were photocopied and returned to the patients. Collected prescriptions were assessed for patient's information, doctor's information and their legibility using predefined criteria by a calibrated examiner. The collected data was analyzed and presented as percentage and frequencies. Results: All the collected prescriptions were lacking one or more aspects of patient's as well as doctor's details. About $23.4 \%$ prescriptions had poor legibility and $21.3 \%$ prescriptions lacked details about more than one drug. Conclusion: The quality and nature of prescriptions written in Dhule city is substandard and it requires use of a standardized prescription format to be used. Also regarding legibility, computerization can be the answer to the problem.
\end{abstract}

Key words: Medication error, Drug prescription, Prescription error, Writing.

\section{INTRODUCTION}

Prescription writing is one of the core competencies expected from medical fraternity. Prescribing appropriate drugs in correct doses may influence patient's health positively. Decision-making and proper transcribing are essential qualities of writing an ideal prescription. ${ }^{1}$ Proper prescription writing, which is an essential skill for medical practitioners, is the primary intervention that doctors offer to the suffering humanity. ${ }^{2} \mathrm{~A}$ correct prescription should consist of all the necessary elements to ensure a clear and correct communication between a physician and a pharmacist. Any error in writing a proper prescription leads to prescription errors which in turn lead to medication errors. $^{3}$
Normal features of a prescription include identity of the patient and physician, information about the drug such as name, correct dose, form, route, timing, frequency and duration of administration. ${ }^{5}$ A prescription error is a failure in the prescription writing process which consists of incorrect information about one or more of these aspects of a prescription. Incorrect prescription writing can not only lead to medication error but also to wastage of resources, adverse clinical effects and economic loss to the patient. Prescription errors usually involve incorrect medication, incorrect dosage, handwriting legibility, lapse in dosage forms, routes. ${ }^{6}$
DOI: 10.5530/ijopp.10.4.54

Address for correspondence: Mahesh R. Khairnar, Department of Public Health Dentistry, Bharati Vidyapeeth Deemed University Dental College and Hospital, Sangli, INDIA.

Phone no: +91-7045653288 Email Id: kmahesh222@gmail. com

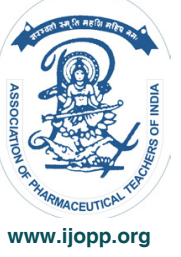


The periodic evaluation of prescriptions can prove to be important to assess the rational use of drugs in terms of prescribing, dispensing and to evaluate the patient understanding about the use of drugs. Prescription patterns and skills of junior doctors and medical students have been reported in the literature. However, literature about prescription writing skills of the practitioners in India is lacking. Hence the present survey was designed with an aim to assess the quality and content of prescriptions provided by the various doctors practicing in Dhule city, Maharashtra, India. The study did not focus on whether the drugs were indicated for the patient's illness, but on the layout and content of the prescription. In particular, the study assessed the quality of the prescriptions in terms of the adequacy and clarity of the information contained therein.

\section{MATERIAL AND METHODS}

The present study was a descriptive cross-sectional survey conducted to assess prescription writing skills of doctors practicing in Dhule city. Ethical Approval was obtained from Institutional Review Board of ACPM Dental College and Hospital, Dhule. The selected pharmacy shop owners were explained about the purpose of the study and permission was obtained to photocopy the prescription.

\section{Sample size determination}

A pilot study was conducted on 15 prescriptions to determine the sample size. The prevalence of prescription errors among those 15 prescriptions was found to be approximately $59 \%$. The sample size was determined by fixing probability of committing type I error $(\alpha)$ at $5 \%$ and that of type II error ( $\beta$ ) at $20 \%$ thus maintaining the power at $80 \%$. The minimum sample size obtained was 278. A total of 286 prescription samples were obtained during actual study.

5 pharmacy shops in Dhule city were randomly selected by lottery dip technique for present study. All the prescriptions which were carried to the selected pharmacy shop by patients/others on selected days were included in the study. Prescriptions written on the scrap of paper were excluded.

\section{Method of data collection}

A detailed schedule of the study was prepared well in advance. As per the schedule, collection of prescriptions at selected pharmacies was done for 5 consecutive days in month of October 2017. The prescriptions obtained therein were photocopied and returned to the patients.
The prescriptions were checked for patient's information, doctor's information, drug details and its legibility using pre-defined criteria. ${ }^{7}$ A single investigator was calibrated to reduce the examiner variability prior to the start of the study. Test retest reliability was assessed on 15 prescriptions and kappa value obtained was 0.78 .

\section{Prescribing criteria format}

A pre-defined prescription format was used for assessment which consisted of following parameters:

1. Patient information: Name, Age, Gender, Address, Contact number

2. Doctor information: Name, Degree, Registration number, Contact number, Signature

3. Clarity of prescription was assessed based on legibility, which was assessed on a 4-point rating scale system:

- Score 1: Prescription details are clear and legible

- Score 2: Clear but requires effort to read

- Score 3: One aspect not clear (patient name/ drug name)

- Score 4: More than one aspect not clear

4. Details of the drug prescribed were assessed on a 4-point rating scale system:

- Score 1: Clear and legible, Drug details present

- Score 2: Clear but requires effort to read

- Score 3: Criteria not met for one drug

- Score 4: Criteria not met for more than one drug

An overall rating system based on the above criteria was also given:

Scoring: Parameter present (1), absent (0)

Legibility: $1-4$

Drug details: 1-4

\section{Statistical analysis}

The data was compiled using Microsoft excel spreadsheet 2010 and was subjected to statistical analysis using SPSS version 17 (SPSS Inc, Chicago, USA). The data is 
presented in frequencies and percentage.

\section{RESULTS}

A total of 286 prescriptions were procured for the actual study. Out of 286 prescriptions obtained, 266 (93\%) had patients' name mentioned, whereas only $114(39.9 \%)$ prescription had patients' age mentioned in them. Only $59(20.6 \%)$ prescriptions had patients gender mentioned in them. Patients' contact details i.e. address, and contact no. were mentioned only in 75 $(26.2 \%)$ and $24(8.4 \%)$ prescriptions respectively (Table 1). Regarding doctors' information, 269 (94.1\%) and 263 $(92 \%)$ prescriptions contained doctors' name and degree respectively. Doctors' registration number was mentioned only in 193 (67.5\%) prescriptions. Only 190 $(66.4 \%)$ prescriptions were signed by doctors. However, $262(91.6 \%)$ prescriptions had doctors' contact number mentioned (Table 2). On assessing the legibility of the prescriptions on rating scale, $67(23.4 \%)$ prescriptions found to have score 4 attributing to major prescription errors. 33 (11.5\%) prescriptions had score 3, 75 (26.2\%) had score 2, whereas 111 (38.8\%) prescriptions had score 1 (Table 3). 61 (21.3\%) prescriptions lacked details about more than one drug (Table 4).

\section{DISCUSSION}

A prescription is a health-care tool employed by a physician or other qualified health care practitioner in the form of instructions that govern the plan of care for an individual patient. The term often refers to a health care provider's written authorization for a patient to purchase a prescription drug from a pharmacist. Prescriptions may be entered into an electronic medical record system and transmitted electronically to a pharmacy or it may be handwritten on preprinted prescription forms. In some cases, a prescription may be transmitted from the physician to the pharmacist orally by telephone; however, this practice may increase the risk of medical error. The content of a prescription includes the name and address of the prescribing provider and of the patient and any other legal requirement, specific treatment prescribed and an authorized signature. It constitutes a way of communication between a caregiver and a pharmacist. Proper prescription writing is an important aspect and serves as primary intervention that physician offers to the suffering humanity. Hence, prescribing is an important feature of healthcare which if not done carefully may harm the patient. ${ }^{6}$

The results of this study have thrown light on some important facts. In most prescriptions, one or more

\begin{tabular}{ccc}
\multicolumn{2}{l}{$\begin{array}{l}\text { Table 1: Patient details: percentage of prescriptions } \\
\text { showing missing information. }\end{array}$} \\
\hline Patient details & $\begin{array}{c}\text { Mentioned } \\
\mathbf{n}(\%)\end{array}$ & $\begin{array}{c}\text { Not mentioned } \\
\mathbf{n}(\%)\end{array}$ \\
\hline Name & $266(93)$ & $20(7)$ \\
Age & $114(39.9)$ & $172(60.1)$ \\
Gender & $59(20.6)$ & $227(79.4)$ \\
Address & $75(26.2)$ & $211(73.8)$ \\
Contact no & $24(8.4)$ & $262(91.6)$ \\
\hline
\end{tabular}

Table 2: Doctor's details: percentage of prescriptions showing missing information.

\begin{tabular}{ccc} 
Doctor details & $\begin{array}{c}\text { Mentioned } \\
\mathbf{n}(\%)\end{array}$ & $\begin{array}{c}\text { Not mentioned } \\
\mathbf{n}(\%)\end{array}$ \\
\hline Name & $269(94.1)$ & $17(5.9)$ \\
Degree & $263(92)$ & $23(8)$ \\
Reg No & $193(67.5)$ & $93(32.5)$ \\
Dated Signature & $190(66.4)$ & $96(33.6)$ \\
Contact no & $262(91.6)$ & $24(8.4)$ \\
\hline
\end{tabular}

Table 3: Legibility of the samples could be noted as follows: (4-point scale).

\begin{tabular}{lc}
\multicolumn{1}{c}{ Score } & $\mathbf{n}(\%)$ \\
\hline 1. Prescription details are clear and legible & $111(38.8)$ \\
2. Clear but requires effort to read & $75(26.2)$ \\
3. One aspect not clear (patient name/drug name) & $33(11.5)$ \\
4. More than one aspect not clear & $67(23.4)$ \\
\hline
\end{tabular}

Table 4: Drug details of the samples could be noted as follows: (4-point scale).

\begin{tabular}{lc}
\multicolumn{1}{c}{ Score } & $\mathbf{n}(\%)$ \\
\hline 1. Clear and legible, Drug details present & $122(42.7)$ \\
2. Clear but requires effort to read & $61(21.3)$ \\
3. Criteria not met for one drug & $42(14.7)$ \\
4. Criteria not met for more than one drug & $61(21.3)$ \\
\hline
\end{tabular}

aspects of the patient's personal details were missing (Table 1). In most cases, the concerned doctor's details were also lacking (Table 2). The legibility of the prescriptions was also doubtful in many cases (Table 3 and 4). This may create problems in maintaining proper records and many medico-legal complications may arise. From the patient's point of view, he will not be in a position to intimate the doctor concerned if a particular brand of medicine is not available. This puts the responsibility of selecting a proper substitute on the shoulders of the pharmacist.

Among the prescription errors found in the study, most common error was regarding patient information. 
Such errors occur because of professional negligence, inadequate knowledge of prescription writing, lack of support staff, doing multiple tasks, tiring or busy schedule. similar results were obtained in the study conducted by Nagesh et al. ${ }^{3}$ and Rathnam et al. ${ }^{7}$ where patient's information accounted for approximately 75\% and $50 \%$ error respectively. Patient identity and contact information are important to ensure that correct patient receives correct medicine and for maintaining patient records also. Also, this information will help pharmacist to contact patient in case of any dispensing error. Next most common error was regarding doctor's registration number $(32.5 \%)$ and doctor's signature $(33.6 \%)$. These two parameters have been made mandatory by Medical Council of India ${ }^{8}$ and Food and Drug Administration, Maharashtra State, India. ${ }^{9}$ Doctor's name and contact information were missing from 5.9\% and $8.4 \%$ prescriptions respectively. Doctor's identity and contact info are essential on prescription to ensure timely approach by the pharmacist to the physician for clarification in case of any doubtful prescription.

In the present study, errors related to legibility of prescription were also seen. About $23.4 \%$ prescription lacked legibility for more than one aspect of patient/ doctor information. About $21.3 \%$ prescription lacked clarity and legibility regarding drug details. This is in conformity with the findings of Mendonca $e t$ al. where $26 \%$ prescription lacked clarity and legibility and were classified as having low legibility or being illegible. ${ }^{10}$ The results obtained in the study conducted by Nagesh $e t$ al., $37.3 \%$ and $52 \%$ prescription lacked legibility for more than one aspect of patient/ doctor information and clarity and legibility in regard to drug details respectively. ${ }^{3}$ Inaccuracy or incompleteness in prescribing or poor handwriting can lead to misinterpretation by pharmacist and can result in errors in drug dispensing. This may also pose a problem in maintaining proper records. Difficult to read handwriting and incomplete medication orders can decrease the efficiency of care and can increase the risk of medication error. ${ }^{10}$

These errors can be minimized by use of pre-printed prescriptions, training assistant staffs who write the prescriptions and informing each patient about the name of the drug and purpose of prescribing it. Patients should also be encouraged to bring the medications purchased to show them to the doctor to identify potential errors. The ideal solution to reduce such errors is digitalization. A well formatted and properly worded prescription format can be used by all the doctors. This will also allow them the flexibility of highlighting the instructions which are already printed on the prescription. Computerized prescription will also allow for maintaining proper record of the medicine which patient has been taking. Computerized prescriptions would ensure legibility and completeness of the prescriptions and would help prevent errors like ordering errors, wrong dose. The findings of the present study along with previously conducted studies highlight the disparity in the nature and content of the layout of the prescriptions throughout India. There is a need to use a standardized ideal prescription format throughout the country.

\section{CONCLUSION}

It can be seen from the present study that most of the prescriptions screened were incomplete with one or more aspects missing and some of the prescriptions had poor legibility. Hence it can be concluded that a clear policy about the standard of prescriptions and periodic internal monitoring is the answer for quality prescriptions.

\section{SOURCE OF FUNDING}

The authors did not receive funding from any other funding source for this work.

\section{ACKNOWLEDGMENT}

The authors would like to thank pharmacy shop owners for co-operating to conduct the study. The authors are also thankful to the patients or others who brought the prescriptions to pharmacy shops and gave the permission to photocopy those prescriptions.

\section{CONFLICT OF INTEREST}

The authors declare that this research does not have any conflict of interest with anyone or any Institute.

\section{ABBREVIATION USED}

SPSS: Statistical Package for the Social Sciences.

\section{SUMMARY}

The study focuses on prescription writing skills of doctors practicing in Dhule city of Maharashtra state, India. Data regarding knowledge about prescription writing among doctors was lacking which was evident from poorly written prescriptions.

Indian Journal of Pharmacy Practice, Vol 10, Issue 4, Oct-Dec, 2017 


\section{REFERENCES}

1. Sudha MJ, Viveka S, Remya S. Assessment of prescription writing skills among undergraduate medical students. Int J Basic Clin Pharmacol. 2016;5(4):1586-93.

2. Maxwell S, Walley $\mathrm{T}$. Teaching safe and effective prescribing in UK Medical schools: a core curriculum for tomorrow's doctors. $\mathrm{Br} \mathrm{J}$ Clin Pharmacol. 2003;55(6):496-503.

3. Nagesh L, Wadgave U, Usha GV. Prescription writing skills of doctors practicing in Davangere city: A cross sectional survey. Indian J Stomatol. 2014;5(2):41-4.

4. Dean B, Schachter M, Vincent $C$, Barber N. Causes of prescribing errors in hospital inpatients: a prospective study. Lancet. 2002;359(9315):1373-8.

5. Velo GP, Minuz P. Medication errors: prescribing faults and prescription errors. Br J Clin Pharmacol. 2009;67(6):624-8.

6. Kumar J, Shaikh M, Kathi MC, Chetty MS, Deka A. Appraisal of Prescription writing skills of preclinical undergraduate students in a medical institute of Nepal. Journal of College of Medical Sciences - Nepal. 2012;6(4):7-13.
7. Rathnam A, Madan N. "Rx - The mistakes we make!!": A short study. Indian J Dent Res. 2011;22(5):684-7

8. Indian Medical Council (Professional Conduct, Etiquette and Ethics) Regulations. Chapter I. 1. Code of medical ethics. 1.4 Display of registration numbers [Online]. 2002 [cited 2017 Nov 2]. Available from: URL: http://www. mciindia.org/RulesandRegulations/CodeofMedicalEthicsRegulations2002. aspx.

9. Food and Drug Administration, Maharashtra State. Maharashtra Model Medicine Prescription Format [Online]. [cited 2017 Nov 2]. Available from: URL: https://fda.maharashtra.gov.in/downloads/fda\%2027feb2014\%20final.pdf.

10. Mendonça JM, Lyra DP, Rabelo JS, Siqueira JS, Balisa-Rocha BJ, Gimenes $\mathrm{FR}$, et al. Analysis and detection of dental prescribing errors at primary health care units in Brazil. Pharm World Sci. 2010;32(1):30-5. 